\title{
FUNCTIONS AND EQUATIONS IN CLASSES OF DISTRIBUTIVE LATTICES WITH PSEUDOCOMPLEMENTATION
}

\author{
by R. BEAZER \\ (Received 24th August 1973)
}

\section{Introduction}

In (8), R. L. Goodstein gave necessary and sufficient conditions for the solvability of equations over distributive lattices with 0 and 1 together with an algorithm for computing a solution whenever one exists. In addition, the same problem was considered for a special class of equations over distributive lattices with pseudocomplementation. The validity of several of Goodstein's results for distributive lattices without 0 and 1 was pointed out by Rudeanu in (15) and (16).

The purpose of this paper is to show that many of the above-mentioned results can be extended to a much larger class of functions and equations over various equational classes of distributive lattices with pseudocomplementation. At the same time we generalise some results of Grätzer (9) and (10) concerning functions on distributive lattices and Boolean algebras satisfying the congruence substitution property.

Finally, a solution of the word problem for the class of distributive lattices with pseudocomplementation is given.

\section{Preliminaries}

Throughout, the reader is assumed to be familiar with the results in (8) and is referred to (11) for the basic lattice theory. We denote the categories of distributive lattices with 0 and 1 , distributive lattices with pseudocomplementation, Stone algebras, Heyting algebras (pseudo-Boolean algebras) and Boolean algebras by $\mathscr{D}_{0,1}, \mathscr{B}_{\omega}, \mathscr{B}_{1}, \mathscr{H}$, and $\mathscr{B}_{0}$ respectively. The algebra in $\mathscr{B}_{\omega}$ obtained from the $2^{n}$-element Boolean algebra by adjoining a new unit will be denoted by $\mathscr{C}_{n}$. The $n$-element chain $\{0,1, \ldots, n-1\}$ will be denoted by $n$.

\section{Functions with the congruence substitution property}

If $\mathscr{A}$ is a class of similar algebras and $A \in \mathscr{A}$ then a function $f: A^{m} \rightarrow A$ is said to be algebraic if there exists a sequence of $m$-ary functions $f_{1}, f_{2}, \ldots, f_{r}$, where $f_{r}=f$ and each $f_{i}(i=1, \ldots, r)$ is either a constant function

$$
a\left(x_{1}, \ldots, x_{m}\right)=a
$$

(where $a$ is a fixed element of $A$ ), an identity function $I_{k}\left(x_{1}, \ldots, x_{m}\right)=x_{k}$ or is 
obtained from preceding functions in the sequence by applying one of the basic operations of the algebra $A$.

A function $f: A^{m} \rightarrow A$ is said to have the congruence substitution property if the implication:

$$
x_{i} \equiv y_{i}(\theta)(i=1,2, \ldots, m) \Rightarrow f\left(x_{1}, \ldots, x_{m}\right) \equiv f\left(y_{1}, \ldots, y_{m}\right)(\theta)
$$

holds for every congruence relation $\theta$ on $A$.

Clearly, every algebraic function has the congruence substitution property. Gratzer (9) has shown that the congruence substitution property characterises the algebraic functions on a Boolean algebra. The same is also true for Post algebras (see (4)). However, it is not true that every function on any distributive lattice with 0 and 1 which has the congruence substitution property is algebraic (see (10)) nor is it true for $p$-rings (see (12)). We give an example, at the end of this section, of a function on a distributive lattice with pseudocomplementation which has the congruence substitution property but is not algebraic.

The following theorems generalise some results of Goodstein (8).

Theorem 3.1. If $L \in \mathscr{B}_{\omega}$ and $f: L^{m} \rightarrow L$ has the congruence substitution property then $f=0$ identically if and only if $\bigvee_{2^{m}} f\left(i_{1}, \ldots, i_{m}\right)=0$, where the join $\bigvee_{2^{m}}$ is taken over all ordered m-tuplets $\left(i_{1}, \ldots, i_{m}\right) \in \mathbf{2}^{m}$.

Proof. It is well-known that if $L \in \mathscr{B}_{\omega}$ then the mapping $x \rightarrow \bar{x}=x^{* *}$ is a homomorphism (in fact, a retraction) from $L$ on to the Boolean algebra $S(L)=\left\{x^{*}: x \in L\right\}$. Therefore, since $f$ has the congruence substitution property, we can define a function $\bar{f}: S^{m}(L) \rightarrow S(L)$ by

$$
\bar{f}\left(\bar{x}_{1}, \ldots, \bar{x}_{m}\right)=\overline{f\left(x_{1}, \ldots, x_{m}\right)} .
$$

Besides being well-defined, $f$ also has the congruence substitution property; because if $\bar{\theta}$ is any congruence on $S(L)$ (regarded as a member of $\mathscr{B}_{\omega}$ ) then there exists a congruence $\theta$ on $L$ such that $a \equiv b(\theta)$ if and only if $\bar{a} \equiv \bar{b}(\bar{\theta})$. Of course, any congruence on $S(L)$, regarded as a member of $\mathscr{B}_{\omega}$, is a congruence on $S(L)$ regarded as a member of $\mathscr{B}_{0}$ and visa versa. Therefore, by Gratzer's afore-mentioned theorem, $\vec{f}$ is an algebraic function on the Boolean algebra $S(L)$. Now, $f\left(a_{1}, \ldots, a_{m}\right)=0$ if and only if $f\left(\bar{a}_{1}, \ldots, \bar{a}_{m}\right)=0$ so that $x_{i}=a$ $(i=1,2, \ldots, m)$ is a solution of the equation $f\left(x_{1}, \ldots, x_{m}\right)=0$ in $L$ if and only if $z_{i}=\bar{a}_{i}(i=1,2, \ldots, m)$ is a solution of the equation $\bar{f}\left(z_{1}, \ldots, z_{m}\right)=0$ in $S(L)$. Finally, $\bar{f}=0$ identically in $S(L)$ if and only if $\bigvee_{2^{m}} \bar{f}\left(i_{1}, \ldots, i_{m}\right)=0$ which is equivalent to $\overline{f\left(i_{1}, \ldots, i_{m}\right)}=0$, for all $\left(i_{1}, \ldots, i_{m}\right) \in \mathbf{2}^{m}$, and therefore to

$$
\bigvee_{2^{m}} f\left(i_{1}, \ldots, i_{m}\right)=0
$$


Theorem 3.2. If $L \in \mathscr{B}_{\omega}$ and $f: L^{m} \rightarrow L$ has the congruence subsitution property then the equation $f\left(x_{1}, \ldots, x_{m}\right)=0$ has a solution if and only if

$$
\bigwedge_{2 m} f\left(i_{1}, \ldots, i_{m}\right)=0
$$

When this condition is satisfied, a solution is

$$
x_{j}=\bigwedge_{2^{m-j}} f\left(x_{1}, \ldots, x_{j-1}, 0, i_{j+1}, \ldots, i_{m}\right) .
$$

Proof. The equation $f\left(x_{1}, \ldots, x_{m}\right)=0$ over $L$ has a solution if and only if the auxiliary Boolean equation $\vec{f}\left(z_{1}, \ldots, z_{m}\right)=0$ over $S(L)$ has a solution. A necessary and sufficient condition for the solvability of the auxiliary equation is $\bigwedge_{2^{m}} f\left(i_{1}, \ldots, i_{m}\right)=0$, which, since

$$
\left.\overline{\bigwedge_{2^{m}} f\left(i_{1}, \ldots, i_{m}\right)}=\bigwedge_{2^{m}} \overline{f\left(i_{1}, \ldots, i_{m}\right.}\right)=\bigwedge_{2^{m}} f\left(i_{1}, \ldots, i_{m}\right),
$$

is equivalent to $\bigwedge_{2^{m}} f\left(i_{1}, \ldots, i_{m}\right)=0$. However, when the auxiliary Boolean equation has a solution, a particular solution is

$$
z_{j}=\bigwedge_{2^{m}-j} f\left(z_{1}, \ldots, z_{j-1}, \overline{0}, i_{j+1}, \ldots, i_{m}\right) \quad(j=1, \ldots, m) .
$$

It follows, since

$\bar{x}_{j}=\bigwedge_{2^{m-j}} \overline{f\left(x_{1}, \ldots, x_{j-1}, 0, i_{j+1}, \ldots, i_{m}\right)}=\bigwedge_{2^{m} j} f\left(\bar{x}_{1}, \ldots, \bar{x}_{j-1}, \overline{0}, i_{j+1}, \ldots, i_{m}\right)$,

that $z_{j}=\bar{x}_{j}(j=1, \ldots, m)$ is a solution of the Boolean equation $\bar{f}\left(z_{1}, \ldots, z_{m}\right)=0$ over $S(L)$ and therefore $x_{j}(j=1, \ldots, m)$ is, indeed, a solution of the equation $f\left(x_{1}, \ldots, x_{m}\right)=0$ over $L$.

Remarks. Theorems 3.1 and 3.2 hold for functions with the congruence substitution property over pseudocomplemented semilattices, implicative semilattices and Heyting algebras. All that is necessary is to point out that the crucial mapping $x \rightarrow \bar{x}$ is a homomorphism in any of these categories.

If $L \in \mathscr{B}_{1}$ and $f: L^{m} \rightarrow L$ has the congruence substitution property then by the general solution of the equation $f\left(x_{1}, \ldots, x_{m}\right)=0$ over $L$ we mean a set of $m$ functions with the congruence substitution property,

$$
x_{k}=f_{k}\left(c_{1}, \ldots, c_{m}\right) \quad(k=1, \ldots, m)
$$

such that $f\left(f_{1}, \ldots, f_{m}\right)$ is identically zero and such that if $x_{k}=x_{k}^{0}(k=1, \ldots, m)$ is any particular solution of the equation then there are values $c_{1}^{0}, \ldots, c_{m}^{0}$ of the parameters $c_{1}, \ldots, c_{m}$ for which $x_{k}^{0}=f_{k}\left(c_{1}^{0}, \ldots, c_{m}^{0}\right)$.

Theorem 3.3. If $L \in \mathscr{B}_{1}, f: L^{m} \rightarrow L$ has the congruence substitution property and the equation $f\left(x_{1}, \ldots, x_{m}\right)=0$ over $L$ has a particular solution

then the general solution is

$$
x_{k}=x_{k}^{\dagger}(k=1, \ldots, m)
$$

$$
x_{k}=\left\{x_{k}^{\dagger} \wedge f\left(c_{1}, \ldots, c_{m}\right)\right\} \vee\left\{c_{k} \wedge f^{*}\left(c_{1}, \ldots, c_{m}\right)\right\} \quad(k=1, \ldots, m) .
$$


Proof. It is well known that the identities $\overline{x^{*}}=(\bar{x})^{*},(x \vee y)^{*}=x^{*} \wedge y^{*}$ and $(x \wedge y)^{*}=x^{*} \vee y^{*}$ hold in any Stone algebra. Therefore,

$$
\begin{aligned}
& \bar{x}_{k}=\overline{\left\{x_{k}^{\dagger}\right.} \wedge \overline{\left.f\left(c_{1}, \ldots, c_{m}\right)\right\}} \vee \overline{\left\{c_{k} \wedge f^{*}\left(c_{1}, \ldots, c_{m}\right)\right\}} \\
& \left.=\overline{\left\{x_{k}^{\dagger}\right.} \wedge \bar{f}\left(\bar{c}_{1}, \ldots, \bar{c}_{m}\right)\right\} \vee\left\{\bar{c}_{k} \wedge \bar{f}^{*}\left(\bar{c}_{1}, \ldots, \bar{c}_{m}\right)\right\}
\end{aligned}
$$

where $\bar{f}$ is the auxiliary Boolean function over $S(L)$. Since $x_{k}^{\dagger}(k=1, \ldots, m)$ is a particular solution of the equation $f\left(x_{1}, \ldots, x_{m}\right)=0$ over $L$,

$$
z_{k}=\overline{x_{k}^{\dagger}}(k=1, \ldots, m)
$$

is a particular solution of the auxiliary equation $\bar{f}\left(z_{1}, \ldots, z_{m}\right)=0$ over $S(L)$. It follows from this and Theorem A of (8) that $z_{k}=\bar{x}_{k}(k=1, \ldots, m)$ is a solution of the Boolean equation $f\left(z_{1}, \ldots, z_{m}\right)=0$ for all $c_{1}, \ldots, c_{m} \in L$. Furthermore, if $x_{k}=x_{k}^{0}(k=1, \ldots, m)$ is any particular solution of the equation over $L$, then $x_{k}^{0}$ is given by the values $c_{k}=x_{k}^{0}$ of the parameters $c_{k}(k=1, \ldots, m)$.

Unlike the case for Boolean algebras, it is not true that every equation over any algebra $L \in \mathscr{B}_{\omega}$ can be written in the form $f=0$. However, every equation is equivalent to an inclusion $f \leqq g$. Furthermore, since $f \leqq g^{*}$ if and only if $f \wedge g^{* *}=0$ in any Stone algebra, Theorems 3.1-3.3 are effective in dealing with solutions of any inequality of the form $f \leqq g^{*}$.

With these remarks in mind we prove the following:

Theorem 3.4. If $L \in \mathscr{B}_{1}$ has a smallest dense element $d_{0}$ and $f, g: L^{m} \rightarrow L$ have the congruence substitution property then $f=g$ identically if and only if

$$
f\left(\alpha_{1}, \ldots, \alpha_{m}\right)=g\left(\alpha_{1}, \ldots, \alpha_{m}\right)
$$

holds whenever $\alpha_{i} \in\left\{0, d_{0}, 1\right\}(i=1, \ldots, m)$.

Proof. Suppose the functions $f$ and $g$ agree on the set $\left\{0, d_{0}, 1\right\}$ but

$$
a=f\left(a_{1}, \ldots, a_{m}\right) \neq g\left(a_{1}, \ldots, a_{m}\right)=b
$$

for some $a_{j} \in L(j=1, \ldots, m)$. Now, since any $L \in \mathscr{B}_{1}$ is a subdirect product of copies of $\mathscr{C}_{0}$ and $\mathscr{C}_{1}$, either there exists a homomorphism $\phi_{0}$ from $L$ on to $\mathscr{C}_{0}$ such that $\phi_{0}(a) \neq \phi_{0}(b)$ or there exists a homomorphism $\phi_{1}$ from $L$ on to $\mathscr{C}_{1}$ such that $\phi_{1}(a) \neq \phi_{1}(b)$. In this first case, since $\phi_{0}(0)$ and $\phi_{0}(1)$ are the only elements of $\mathscr{C}_{0}$, we have $f_{0}=g_{0}$ identically, where $f_{0}: \mathscr{C}_{0}^{m} \rightarrow \mathscr{C}_{0}$ is defined by

$$
f_{0}\left(\phi_{0}\left(x_{1}\right), \ldots, \phi_{0}\left(x_{m}\right)\right)=\phi_{0}\left(f\left(x_{1}, \ldots, x_{m}\right)\right)
$$

and $g_{0}: \mathscr{C}_{0}^{m} \rightarrow \mathscr{C}_{0}$ is defined similarly. This, of course, is contrary to

$$
\phi_{0}(a) \neq \phi_{0}(b) \text {. }
$$

We deal with the second case by first showing that $\phi_{1}\left(d_{0}\right)$ is the (unique) dense element in the three-element chain $\mathscr{C}_{1}$ distinct from the greatest element 1. 
If, to the contrary, $\phi_{1}\left(d_{0}\right)=1$ then, since $d_{0}$ is the smallest dense element in $L$, $\phi_{1}(d)=1$ for all dense elements $d$ in $L$. It follows, since $x=x^{* *} \wedge\left(x \vee x^{*}\right)$ and $x \vee x^{*}$ is dense for all $x \in L$, that

$$
\phi_{1}(x)=\phi_{1}\left(x^{* *}\right) \wedge \phi_{1}\left(x \vee x^{*}\right)=\phi_{1}\left(x^{* *}\right)=\left\{\phi_{1}(x)\right\}^{* *}
$$

for all $x \in L$. This yields, since $\phi_{1}$ is surjective, an obvious contradiction. Finally, since $\phi_{1}(0), \phi_{1}\left(d_{0}\right)$ and $\phi_{1}(1)$ are the only elements in $\mathscr{C}_{1}$, we see that $f_{1}=g_{1}$ identically on $\mathscr{C}_{1}$, where $f_{1}: \mathscr{C}_{1}^{m} \rightarrow \mathscr{C}_{1}$ is defined by

$$
f_{1}\left(\phi_{1}\left(x_{1}\right), \ldots, \phi_{1}\left(x_{m}\right)\right)=\phi_{1}\left(f\left(x_{1}, \ldots, x_{m}\right)\right)
$$

and $g_{1}: \mathscr{C}_{1}^{m} \rightarrow \mathscr{C}_{1}$ is defined similarly. This, of course, contradicts $\phi_{1}(a) \neq \phi_{1}(b)$ and the proof is complete.

Corollary 3.5. If $B \in \mathscr{B}_{0}$ and $f, g: B^{m} \rightarrow B$ have the congruence substitution property then $f=g$ identically if and only if $f\left(i_{1}, \ldots, i_{m}\right)=g\left(i_{1}, \ldots, i_{m}\right)$ for all $\left(i_{1}, \ldots, i_{m}\right) \in 2^{m}$.

Stone algebras are well known to form an equational class. The class of Stone algebras having a smallest dense element may be regarded as an equational class of algebras of the form $\mathscr{L}=\langle L ; \vee, \wedge ; * ; 0,1, d\rangle$ where

$$
\langle L ; \vee, \wedge ; * ; 0,1\rangle
$$

is a Stone algebra, $d^{*}=0$ and $d \wedge\left(x \vee x^{*}\right)=d$ for all $x \in L$.

It follows as a special case of results proved in (6) that a Heyting algebra belongs to the (equational) class $\mathscr{H}_{3}$ of all Heyting algebras satisfying the identity $\bigvee_{i=1}^{3}\left(x_{i} * x_{i+1}\right)=1$ if and only if it is a subdirect product of copies of the two and three element chains $\mathscr{C}_{0}, \mathscr{C}_{1}$. An immediate consequence is the following:

Theorem 3.6. If $H \in \mathscr{H}_{3}$ has a smallest dense element $d_{0}$ and $f, g: H^{m} \rightarrow H$ have the congruence substitution property then $f=g$ identically if and only if $f$ and $g$ agree on the set $\left\{0, d_{0}, 1\right\}$.

The next result generalises some important properties of algebraic functions on Heyting algebras first proved in (13).

Theorem 3.7. If $H \in \mathscr{H}$ and $f: H^{m} \rightarrow H$ has the congruence substitution property then, for $i=1, \ldots, m$,

(i) $a \wedge f\left(x_{1}, \ldots, x_{m}\right)=a \wedge f\left(x_{1}, \ldots, x_{i-1}, a \wedge x_{i}, x_{i+1}, \ldots, x_{m}\right)$

and (ii) $a * f\left(x_{1}, \ldots, x_{m}\right)=a * f\left(x_{1}, \ldots, x_{i-1}, a * x_{i}, x_{i+1}, \ldots, x_{m}\right)$.

Proof. If $a \in H$ then the smallest congruence $\theta_{a, 1}$ on $H$ for which $a$ and 1 belong to the same congruence class can be described by $x \equiv y\left(\theta_{a, 1}\right)$ if and only if $x \wedge a=y \wedge a$. Now, $a \equiv 1\left(\theta_{a, 1}\right)$ implies $x_{i} \equiv a \wedge x_{i}\left(\theta_{a, 1}\right)$ so that

$f\left(x_{1}, \ldots, x_{m}\right) \equiv f\left(x_{1}, \ldots, x_{i-1}, a \wedge x_{i}, x_{i+1}, \ldots, x_{m}\right) \quad\left(\theta_{a, 1}\right)$ 
and therefore (i) holds. Next, $a \equiv 1\left(\theta_{a, 1}\right)$ implies that $a * x_{i} \equiv 1 * x_{i}=x_{i}\left(\theta_{a, 1}\right)$ and so $a \wedge f\left(x_{1}, \ldots, x_{m}\right)=a \wedge f\left(x_{1}, \ldots, x_{i-1}, a * x_{i}, x_{i+1}, \ldots, x_{m}\right)$. It follows, since the identity $a *(a \wedge x)=a * x$ holds in any Heyting algebra, that

$$
a * f\left(x_{1}, \ldots, x_{m}\right)=a * f\left(x_{1}, \ldots, x_{i-1}, a * x_{i}, x_{i+1}, \ldots, x_{m}\right) .
$$

Corollary 3.8. If $H \in \mathscr{H}$ and $f: H \rightarrow H$ has the congruence substitution property then the following hold:

(i) $f(x \vee y) \wedge x=f(x) \wedge x$

(ii) $f(x \vee y) \wedge(x \vee y)=[f(x) \wedge x] \vee[f(y) \wedge y]$

(iii) $f(x \vee y) \wedge\left(x^{*} \vee y^{*}\right)=\left[f(x) \wedge y^{*}\right] \vee\left[f(y) \wedge x^{*}\right]$

(iv) $(x \vee y) * f(x \wedge y)=[x * f(y)] \wedge[y * f(x)]$.

Proof. See (13).

\section{Examples}

We give an example of a function on a distributive lattice with pseudocomplementation (in fact, on a Stone algebra) which has the congruence substitution property but is not algebraic.

The three-element chain $\mathscr{C}_{1}=\{0, p, 1\}$ (regarded as a member of $\mathscr{B}_{\omega}$ ) has exactly three congruences, namely $\omega_{0}, \theta_{p, 1}, \omega_{1}$ where $\omega_{0}, \omega_{1}$ are the trivial congruences and $\theta_{p, 1}$ has congruence classes $\{1, p\},\{0\}$. The function $f: \mathscr{C}_{1} \rightarrow \mathscr{C}_{1}$ defined by $f(0)=1, f(p)=1, f(1)=p$ has the congruence substitution property. We show that $f$ is not algebraic. First, we remark that if $L \in \mathscr{B}_{1}$ and $f: L \rightarrow L$ is algebraic then, by induction on the structure of $f$, it can be shown that $f(x)$ is expressible in the form $(a \wedge x) \vee\left(b \wedge x^{*}\right) \vee\left(c \wedge x^{* *}\right)$. Therefore, if $f$ is algebraic then

$$
(a \wedge p) \vee c=1(=f(p)) \text { and } \quad a \vee c=p(=f(1))
$$

so that $p=p \wedge(p \vee c)=(a \vee c) \wedge(p \vee c)=(a \wedge p) \vee c=1$. Thus $f$ is not algebraic.

Some Stone algebras having a smallest dense element are:

(a) Finite Stone algebras.

(b) Well-ordered sets with greatest elements.

(c) Any distributive lattice with a new zero adjoined.

(d) Equationally compact Stone algebras.

(e) Compact, Hausdorff, topological Stone algebras (see (5)).

$(f)$ Injective Stone algebras (see (11)).

Of the algebras in the above list, examples $(a),(d),(e)$ and $(f)$ are all Heyting algebras. 


\section{Pre-Post algebras and Post algebras}

If $D \in \mathscr{D}_{0,1}$ and $E$ is a finite chain with $n \geqq 2$ elements then we write $[D]_{n}$ for the free product $D * E$ of $D$ and $E$ in the category of distributive lattices with 0 and 1 (and 0, 1-preserving lattice homomorphisms) and call it the pre-Post algebra of order $n$ over $D$. If $B \in \mathscr{B}_{0}$ then $[B]_{n}$ is the Post algebra of order $n$ over $B$ (see e.g. (14)). From the papers of Balbes and Dwinger (1), (2) and Rouseau (14) we have the following equational characterisation of pre-Post algebras :

Theorem 4.1. In order that the algebra

$$
\left\langle L ; \vee, \wedge ; D_{1}, \ldots, D_{n-1} ; e_{0}, \ldots, e_{n-1}\right\rangle
$$

with two binary $(\vee, \wedge), n-1$ unary $\left(D_{1}, \ldots, D_{n-1}\right)$ and $n$ nullary $\left(e_{0}, \ldots, e_{n-1}\right)$ operations should be a pre-Post algebra of order $n$, it is necessary and sufficient that $L \in \mathscr{D}_{0,1}$ and the following identities hold: $(i=1, \ldots, n-1)$

$$
\begin{aligned}
D_{i}(x \vee y) & =D_{i}(x) \vee D_{i}(y) \\
D_{i}(x \wedge y) & =D_{i}(x) \wedge D_{i}(y) \\
D_{i}\left(D_{k}(x)\right) & =D_{k}(x) \\
D_{i}\left(e_{k}\right) & =\left\{\begin{array}{ll}
1, & i \leqq k \\
0, & i>k
\end{array} \quad(k=1, \ldots, n-1)\right. \\
x & =\bigvee_{i \in n} D_{i}(x) \wedge e_{i} .
\end{aligned}
$$

Some properties of pre-Post algebras which we need are stated in the following:

Lemma 4.2. In any pre-Post algebra $L$ of order $n$ the following are true:

(i) The set $E=\left\{e_{0}, \ldots, e_{n-1}\right\}$ of nullary operations on $L$ forms a chain $0=e_{0}<e_{1}<\ldots<e_{n-1}=1$ and is a sublattice of $L$.

(ii) The mappings $D_{i}$ have a common image $D$, which is a sublattice of $L$, and reduce to the identity on $D$.

(iii) $D_{i}(x) \leqq D_{j}(x)$ whenever $j \leqq i(i, j=1, \ldots, n-1)$.

(iv) $x \leqq y \Leftrightarrow D_{i}(x) \leqq D_{i}(y)$ for all $i=1, \ldots, n-1$.

(v) $D_{n-1}(x) \leqq x \leqq D_{1}(x)$.

(vi) $L=D * E\left(=[D]_{n}\right)$.

If $[D]_{n}$ is a pre-Post algebra of order $n$ with chain of constants

$$
E=\left\{e_{0}, \ldots, e_{n-1}\right\}
$$

then a function $f:[D]_{n}^{m} \rightarrow[D]_{n}$ is said to be monotone on $E$ if

$$
f\left(e_{\alpha_{1}}, \ldots, e_{\alpha_{m}}\right) \leqq f\left(e_{\beta_{1}}, \ldots, e_{\beta_{m}}\right)
$$

whenever the elements $\left(\alpha_{1}, \ldots, \alpha_{m}\right),\left(\beta_{1}, \ldots, \beta_{m}\right) \in n^{m}$ satisfy

$$
\left(\alpha_{1}, \ldots, \alpha_{m}\right) \leqq\left(\beta_{1}, \ldots, \beta_{m}\right) \text {. }
$$


Using the preceding results we can generalise, to the class of pre-Post algebras, some results of Gratzer (10) about functions on distributive lattices with 0 and 1 which satisfy the congruence substitution property. The proofs are slight variations of the proofs of related results given in (4) and are therefore omitted.

Theorem 4.3. If $[D]_{n}$ is a pre-Post algebra of order $n$ and $f, g:[D]_{n}^{m} \rightarrow[D]_{n}$ have the congruence substitution property then $f=g$ identically if and only if $f$ and $g$ agree on the chain of constants of $[D]_{n}$.

Theorem 4.4. If $[D]_{n}$ is a pre-Post algebra of order $n$ and $f:[D]_{n}^{m} \rightarrow[D]_{n}$ has the congruence substitution property then $f$ is algebraic if and only if it is monotone on the chain of constants of $[D]_{n}$.

The following canonical form for algebraic functions on pre-Post algebras is a straight forward generalisation of the canonical form for algebraic functions on distributive lattices with 0 and 1 obtained in (8).

Theorem 4.5. If $[D]_{n}$ is a pre-Post algebra of order $n$ and $f:[D]_{n}^{m} \rightarrow[D]_{n}$ is algebraic then

$$
f\left(x_{1}, \ldots, x_{m}\right)=\bigvee_{n^{m}} f\left(e_{\alpha_{1}}, \ldots, e_{\alpha_{m}}\right) \wedge \bigwedge_{k=1}^{m} D_{\alpha_{k}}\left(x_{k}\right),
$$

where the join $\bigvee_{n^{m}}$ is taken over all $\left(\alpha_{1}, \ldots, \alpha_{m}\right) \in \boldsymbol{n}^{m}$.

In (14) it was shown that an algebra

$$
\left\langle L ; \vee, \wedge, * ; D_{1}, \ldots, D_{n-1} ; e_{0}, \ldots, e_{n-1}\right\rangle
$$

is a Post algebra of order $n$ if and only if $H=\langle L ; \vee, \wedge, *\rangle \in \mathscr{H}$ and the identities listed in Theorem 4.1 together with the identity $D_{1}(x) \vee D_{1}^{*}(x)=1$ hold. The common image of the mappings $D_{i}$ is a Boolean subalgebra of $H$ and $L=B * E\left(=[B]_{n}\right)$.

In any Post algebra $[B]_{n}$ it is known that the elements $C_{i}(x) \in B$ defined by

$$
C_{0}(x)=D_{1}^{*}(x), C_{n-1}(x)=D_{n-1}(x)
$$

and $C_{i}(x)=D_{i}(x) \wedge D_{i+1}^{*}(x)(i=1, \ldots, n-2)$ form a partition of 1 in that $C_{i}(x) \wedge C_{j}(x)=0 \quad(i \neq j)$ and $\bigvee_{i \in n} C_{i}(x)=1$. Furthermore, every element $x \in[B]_{n}$ has a partition representation $x=\bigvee_{i \in n} C_{i}(x) \wedge e_{i}$, which is unique in the sense that if $x=\bigvee_{i \in n} a_{i} \wedge e_{i}$ and the elements $a_{0}, \ldots, a_{n-1}$ partition 1 , then $a_{i}=C_{i}(x)(i=0, \ldots, n-1)$. In (4) it was shown that if $f:[B]_{n}^{m} \rightarrow[B]_{n}$ has the congruence substitution property and $g:[B]_{n}^{m} \rightarrow[B]_{n}$ is defined by

$$
g\left(x_{1}, \ldots, x_{m}\right)=\bigvee_{n^{m}} f\left(e_{\alpha_{1}}, \ldots, e_{\alpha_{m}}\right) \wedge \bigwedge_{k=1}^{m} C_{\alpha_{k}}\left(x_{k}\right)
$$

then $f=g$ identically. It follows that an algebraic function on $[B]_{n}$ may be thought of as a function built up from the constant functions $a\left(x_{1}, \ldots, x_{m}\right)=a$ 
and the identity functions $I_{k}\left(x_{1}, \ldots, x_{m}\right)=x_{k}$ by a finite sequence of applications of the operations $\vee, \wedge, C_{0}, \ldots, C_{n-1}$.

In (7) Epstein defines a Post function (of $m$-variables) to be a function obtained from the constant functions $E_{k}\left(x_{1}, \ldots, x_{m}\right)=e_{k}(k=0, \ldots, n-1)$ and the identity functions by a finite sequence of the operations $\vee, \wedge, C_{0}, \ldots, C_{n-1}$. The next theorem gives a canonical form for algebraic functions on $[B]_{n}$ which contains the one obtained for Post functions in (7).

Theorem 4.6. If $[B]_{n}$ is a Post algebra of order $n$ and $f:[B]_{n}^{m} \rightarrow[B]_{n}$ is algebraic then

$$
f\left(x_{1}, \ldots, x_{m}\right)=\bigvee_{\mathbf{m}^{m}} f\left(e_{\alpha_{1}}, \ldots, e_{a_{m}}\right) \wedge \bigwedge_{k=1}^{m} C_{\alpha_{k}}\left(x_{k}\right) .
$$

Proof. It follows verbatim from (7, Theorem 13) that the $n^{m}$ fundaments $\bigwedge_{k=1}^{m} C_{\alpha_{k}}\left(x_{k}\right)$ partition 1 and that the theorem is true for the (general) constant functions, the identity functions and the functions $f_{1} \vee f_{2}, f_{1} \wedge f_{2}$ whenever $f_{1}$ and $f_{2}$ satisfy the theorem. If $f$ satisfies the theorem then, since

$$
f\left(e_{\alpha_{1}}, \ldots, e_{\alpha_{m}}\right)=\bigvee_{k \in n} e_{k} \wedge C_{k}\left(f\left(e_{\alpha_{1}}, \ldots, e_{\alpha_{m}}\right)\right)
$$

and $[B]_{n}$ is distributive, it follows that $f\left(x_{1}, \ldots, x_{m}\right)=\bigvee_{k \in n} e_{k} \wedge T_{k}$ where

$$
T_{k}=\bigvee_{n^{m}} C_{k}\left(f\left(e_{\alpha_{1}}, \ldots, e_{\alpha_{m}}\right)\right) \wedge \bigwedge_{l=1}^{m} C_{\alpha l}\left(x_{l}\right) \text {. }
$$

Utilising the distributivity of $[B]_{n}$ and the partition property of elements and fundaments, it is clear that the elements $T_{k}(k=0, \ldots, n-1)$ partition 1 . The uniqueness of the partition representation of $f\left(x_{1}, \ldots, x_{m}\right)$ implies that $T_{k}=C_{k}\left(f\left(x_{1}, \ldots, x_{m}\right)\right)$ and therefore the theorem is true for the function $C_{k} f$. The result follows by induction on the structure of $f$.

Corollary 4.7. If $[B]_{n}$ is a Post algebra of order $n$ and $f, g:[B]_{n}^{m} \rightarrow[B]_{n}$ are algebraic then $f=g$ identically if and only if $f$ and $g$ agree on the chain of constants of $[B]_{n}$.

Theorem 4.8. If $[B]_{n}$ is a Post algebra of order $n$ and $f:[B]_{n}^{m} \rightarrow[B]_{n}$ is algebraic then the equation $f\left(x_{1}, \ldots, x_{m}\right)=0$ has a solution if and only if $\bigwedge_{n^{m}} f\left(e_{\alpha_{1}}, \ldots, e_{\alpha_{m}}\right)=0$.

Proof. It follows, since $f=0$ if and only if $D_{1}(f)=0$ and $D_{1}$ is a lattice homomorphism which reduces to the identity of the underlying Boolean algebra $B$, that the equation $f\left(x_{1}, \ldots, x_{m}\right)=0$ has a solution if and only if the equation $\bigvee_{n^{m}} D_{1}\left(f\left(e_{\alpha_{1}}, \ldots, e_{\alpha_{m}}\right)\right) \wedge \bigwedge_{k=1}^{m} C_{\alpha_{k}}\left(x_{k}\right)=0$ has a solution. The uniqueness of the partition representations for the elements $x_{1}, \ldots, x_{m}$ shows that this is equivalent 
to the simultaneous solvability of the system $\Sigma$ of Boolean equations over $B$ given by

$$
\begin{gathered}
\bigvee_{n^{m}} D_{1}\left(f\left(e_{\alpha_{1}}, \ldots, e_{a_{m}}\right)\right) \wedge \bigwedge_{k=1}^{m} x_{k, \alpha_{k}}=0, \\
\bigvee_{\alpha \in n} x_{k, \alpha}=1, \\
x_{k, \alpha} \wedge x_{k, \beta}=0, \quad(k=1, \ldots, m ; \alpha, \beta \in n, \alpha \neq \beta) .
\end{gathered}
$$

On writing $\psi_{1}\left(x_{1,0}, \ldots, x_{1, n-1} ; \ldots ; x_{m, 0}, \ldots, x_{m, n-1}\right)$ for

$$
\bigvee_{k=1}^{m}\left\{\left(\bigwedge_{\alpha \in n} x_{k, \alpha}^{*}\right) \wedge \bigvee_{\langle\alpha, \beta\rangle \in n}\left(x_{k, \alpha} \wedge x_{k, \beta}\right)\right\}
$$

where the join $\bigvee$ is taken over all pairs $(\alpha, \beta) \in n^{2}$ with $\alpha \neq \beta$ and writing $\langle\alpha, \beta\rangle \in n$

$\psi_{2}\left(x_{1,0}, \ldots, x_{1, n-1} ; \ldots ; x_{m, 0}, \ldots, x_{m, n-1}\right)$ for

$$
\bigvee_{n^{m}} D_{1}\left(f\left(e_{\alpha_{1}}, \ldots, e_{\alpha_{m}}\right)\right) \wedge \bigwedge_{k=1}^{m} x_{k, \alpha_{k}}
$$

the solvability of the system $\Sigma$ over $B$ is equivalent to the solvability of the Boolean equation

$$
\psi\left(x_{1,0}, \ldots, x_{1, n-1} ; \ldots ; x_{m, 0}, \ldots, x_{m, n-1}\right),
$$

where $\psi=\psi_{1} \vee \psi_{2}$. This is equivalent, by Theorem B of (8), to

$$
I=\bigwedge_{2^{m n}} \psi\left(i_{1,0}, \ldots, i_{1, n-1} ; \ldots ; i_{m, 0}, \ldots, i_{m, n-1}\right)
$$

being 0 where the meet $\bigwedge_{2^{m n}}$ is taken over all possible assignments from $2^{m n}$. In such an assignment we call the set $\left\{i_{k, 0}, \ldots, i_{k, n-1}\right\}$ the $k$ th block. Now, for any assignment from $2^{m n}$ in which $i_{k, a}=i_{k, \beta}=1$ for some $k=1, \ldots, m$ and $\alpha, \beta \in n$ with $\alpha \neq \beta$, the value of $\psi_{1}$, and therefore of $\psi$, is 1 . Thus, we restrict attention to those assignments from $2^{m n}$ in which 1 appears at most once in each block. Furthermore, we can restrict attention to those assignments in which 1 appears exactly once in each block; because if, for example, the $k$ th block does not contain 1 then the value of $\bigwedge_{\alpha \in n} x_{k, \alpha}^{*}$ is 1 and therefore so is the value of $\psi$. Clearly there are exactly $n^{m}$ such " basic" assignments from $2^{m n}$. For each basic assignment, the value of $\psi_{1}$ is 0 so that the value of $\psi$ is equal to the value of $\psi_{2}$. Consequently, $I$ is the meet of the values assumed by $\psi_{2}$ under the $n^{m}$ basic assignments. Now, for each basic assignment, the value of the term $D_{1}\left(f\left(e_{\alpha_{1}}, \ldots, e_{\alpha_{m}}\right)\right) \wedge \bigwedge_{k=1}^{m} x_{k, \alpha_{k}}$ in $\psi_{2}$ is 0 , unless the $m$-tuplet $\left(\alpha_{1}, \ldots, \alpha_{m}\right) \in n^{m}$ is such that $i_{k, \alpha_{k}}=1$ for all $k=1, \ldots, m$; in which case its value is

$$
D_{1}\left(f\left(e_{\alpha_{1}}, \ldots, e_{\alpha_{m}}\right)\right) \text {. }
$$


For any pre-assigned $m$-tuplet $\left(\alpha_{1}, \ldots, \alpha_{m}\right) \in n^{m}$ it is clear that there is a basic assignment from $2^{m n}$ for which the value of $\psi_{2}$ is $D_{1}\left(f\left(e_{\alpha_{1}}, \ldots, e_{\alpha_{m}}\right)\right)$. Therefore, $I=\bigwedge_{m^{m}} D_{1}\left(f\left(e_{\alpha_{1}}, \ldots, e_{\alpha_{m}}\right)\right)$ so that the equation $f\left(x_{1}, \ldots, x_{m}\right)=0$ is solvable if and only if $D_{1}\left(\bigwedge_{n^{m}} f\left(e_{\alpha_{1}}, \ldots, e_{\alpha_{m}}\right)\right)=0$, or equivalently $\bigwedge_{n^{m}} f\left(e_{\alpha_{1}}, \ldots, e_{a_{m}}\right)=0$.

Remark. In any Post algebra of order $n, x=y$ if and only if

$$
\bigvee_{i \in n}\left(C_{i}(x)+C_{i}(y)\right) \wedge e_{i}=0,
$$

where $C_{i}(x)+C_{i}(y)$ is the symmetric difference of $C_{i}(x)$ and $C_{i}(y)$ in the Boolean algebra $B$. It follows, therefore, that every equation $f=g$ over a Post algebra can be reduced to one of the form $f=0$.

\section{The word problem for $\mathscr{B}_{\omega}$}

The word problem for the classes $\mathscr{D}_{0,1}$ and $\mathscr{B}_{0}$ have long been solved. Balbes (3) has recently shown that the class of pseudocomplemented semilattices has a solvable word problem.

It is known (see (11)) that the lattice of equational subclasses of $\mathscr{B}_{\omega}$ is a chain $\mathscr{B}_{-1} \subset \mathscr{B}_{0} \subset \mathscr{B}_{1} \subset \ldots \subset \mathscr{B}_{\omega}$ of type $\omega+1$. Furthermore, an algebra belongs to the class $\mathscr{B}_{n}(n<\omega)$ if and only if it is a subdirect product of copies of the algebras $\mathscr{C}_{0}, \ldots, \mathscr{C}_{n}$ while an algebra belongs to $\mathscr{B}_{\omega}$ if and only if it is a subdirect product of copies of algebras obtained from arbitrary Boolean algebras by adjoining a new unit. That the word problem for the class $\mathscr{B}_{n}(n<\omega)$ is solvable follows easily from its subdirect representation theorem.

The $n$-ary word algebra associated with the class $\mathscr{B}_{\omega}$ will be denoted by $W_{n}\left(\mathscr{B}_{\omega}\right)$. If $f \in W_{n}\left(\mathscr{B}_{\omega}\right)$ and $L \in \mathscr{B}_{\omega}$ then we write $f_{L}$ for the polynomial over $L$ induced by $f$. If $f, g \in W_{n}\left(\mathscr{B}_{\omega}\right)$ then by $f=g$ we understand that $f_{L}=g_{L}$ identically in every $L \in \mathscr{B}_{\omega}$. The order of $f \in W_{n}\left(\mathscr{B}_{\omega}\right)$ is the length $O(f)$ of the smallest sequence $f^{(1)}, \ldots, f^{(r)}=f$ where every word $f^{(i)}(i=1, \ldots, \mathrm{r})$ is either the zero word, and identity word or is obtained from preceding words by applying one of the operation symbols $\vee, \wedge, *$.

Theorem 5.1. If $f, g \in W_{n}\left(\mathscr{B}_{m}\right)$ then $f=g$ if and only if $f=g$ identically in every algebra $\mathscr{C}_{m} \in \mathscr{B}_{\omega}$ with $m \leqq 2^{n+O(f)+O(g)}$.

Proof. If $f \neq g$ then there exists an algebra $\langle P ; \vee, \wedge ; *\rangle \in \mathscr{B}_{\omega}$ and $a_{1}, \ldots, a_{n} \in P$ such that $f_{P}\left(a_{1}, \ldots, a_{n}\right) \neq g_{P}\left(a_{1}, \ldots, a_{n}\right)$. Let $f_{P}^{(1)}, \ldots, f_{P}^{(O(f))}=f_{P}$ and $g_{P}^{(1)}, \ldots, g_{P}^{(O(g))}=g_{P}$ be sequences for $f_{P}$ and $g_{P}$. Define

$$
b_{i}=f_{P}^{(i)}\left(a_{1}, \ldots, a_{n}\right)(i=1, \ldots, O(f)), c_{j}=f_{P}^{(j)}\left(a_{1}, \ldots, a_{n}\right)(j=1, \ldots, O(g))
$$

and let $L$ be the sublattice of $P$ generated by the set

$$
\left\{a_{1}, \ldots, a_{n}, b_{1}, \ldots, b_{o(f)}, c_{1}, \ldots, c_{o(g)}\right\} .
$$

E.M.S. $-19 / 2-0$ 
Then $L$ has cardinality at most 2 to the power $2^{n+o(f)+o(g)}$ and is pseudocomplemented. Furthermore, if $x^{0}$ denotes the pseudocomplement of $x$ in $L$ then it is clear that if $x \in L$ and $x^{*} \in L$ then $x^{0}=x^{*}$. From this it follows that $b_{i}=f_{L}^{(i)}\left(a_{1}, \ldots, a_{n}\right)(i=1, \ldots, O(f))$ and $c_{j}=g_{L}^{(j)}\left(a_{1}, \ldots, a_{n}\right)(j=1, \ldots, O(g))$ and therefore $f_{L}\left(a_{1}, \ldots, a_{m}\right) \neq g_{L}\left(a_{1}, \ldots, a_{m}\right)$. Now, suppose that $f=g$ identically in $\mathscr{C}_{m}$ for all $m \leqq 2^{n+O(f)+o(g)}$ but $f \neq g$. Then $f_{L}$ is not identically equal to $g_{L}$ in some algebra $L \in \mathscr{B}_{\omega}$ having cardinality at most 2 to the power $2^{n+O(f)+O(g)}$. It follows from the subdirect product representation of $L$ that $L$ is a subdirect product of a finite number of copies of some of the algebras $\mathscr{C}_{0}, \mathscr{C}_{1}, \mathscr{C}_{2}, \ldots$, say $\mathscr{C}_{m_{1}}, \ldots, \mathscr{C}_{m_{t}}$, where $\left|\mathscr{C}_{m_{t}}\right| \leqq|L| \leqq 2^{2 n+O(f)+O(g)}$ and so $m_{i} \leqq 2^{n+O(f)+O(g)}(1 \leqq i \leqq t)$. Therefore, since $f_{L}$ is not identically equal to $g_{L}$ in $L$, there exists an integer $k(1 \leqq k \leqq t)$ such that $f$ is not identically equal to $g$ in $\mathscr{C}_{m_{k}}$. This, of course, is contrary to hypothesis.

\section{REFERENCES}

(1) R. Balbes and Ph. Dwinger, Coproducts of Boolean algebras and chains with applications to Post algebras, Colloq. Math. 24 (1971), 15-25.

(2) R. Balbes and PH. Dwinger, Uniqueness of representations of a distributive lattice as a free product of a Boolean algebra and a chain, Colloq. Math. 24 (1971), 27-35.

(3) R. BALBes, On free pseudo-complemented and relatively pseudo-complemented semi-lattices, Fund. Math. 78 (1973), 119-131.

(4) R. BeAZER, Some remarks on Post algebras, Colloq. Math. 29 (1974), 167-178.

(5) R. Beazer, Intrinsic topologies on Brouwerian semilattices, Proc. London Math. Soc. 28 (1974), 311-334.

(6) R. BEAZER, Hierarchies of distributive lattices satisfying annihilator conditions, J. London Math. Soc. (to appear).

(7) G. Epstein, The lattice theory of Post algebras, Trans. Amer. Math. Soc. 95 (1960), 300-317.

(8) R. L. Goodstern, The solution of equations in a lattice, Proc. Roy. Soc. Edinburgh Section A 67 (1966/67), 231-242.

(9) G. GRÄTZER, On Boolean functions (Notes on lattice theory III), Rev. Roumaine Math. Pures Appl. 7 (1962), 693-697.

(10) G. Grätzer, Boolean functions on distributive lattices, Acta. Math. Acad. Sci. Hungar. 15 (1964), 195-201.

(11) G. GRÄTZER, Lattice Theory (Freeman, San Francisco, 1971).

(12) A. IsKander, Algebraic functions on p-rings, Colloq. Math. 25 (1972), 37-41.

(13) J. C. C. MCKINSEY and A. TARSKI, On closed elements in closure algebras, Ann. of Math. 47 (1946), 122-162. 
(14) G. Rouseau, Post algebras and pseudo-Post algebras, Fund. Math. 67 (1970), 133-145.

(15) S. Rudeanu, On functions and equations in distributive lattices, Proc. Edinburgh Math. Soc. 16 (1968), 49-54.

(16) S. RudeANU, Correction to the paper "On functions and equations in distributive lattices", Proc. Edinburgh Math. Soc. 17 (1970), 105.

The UnIVERSITY OF Glasgow

Glasgow G12 8QW 